\title{
Novel Error Interpretation in case of Linear Parameter Varying Systems
}

\author{
György Eigner*, József K. Tar ${ }^{\dagger}$, and Levente Kovács* \\ ${ }^{*}$ Research and Innovation Center of Obuda University, \\ Physiological Controls Group, Obuda University, Budapest, Hungary \\ ${ }^{\dagger}$ Research and Innovation Center of Obuda University, \\ Antal Bejczy Center for Intelligent Robotics, Obuda University, Budapest, Hungary \\ Email: eigner.gyorgy@phd.uni-obuda.hu, \{kovacs.levente, tar.jozsef\}@nik.uni-obuda.hu
}

\begin{abstract}
The purpose of this paper is to introduce novel error definitions in the case of Linear Parameter Varying (LPV) systems, which can be used as quality criteria in LPV related modeling and control. The current approach relies on the convexity of the LPV polytopic system by defining the difference in the abstract parameter space. The theoretical approach is demonstrated on a well-known glucose-insulin model used in intensive care.
\end{abstract}

\section{INTRODUCTION}

Linear Parameter Varying systems represent an elegant tool handling nonlinear systems as linear ones based on a time varying parameter vector [1]. The general state space representation of the Linear Time Varying (LTV) systems is the following [1]-[3]:

$$
\begin{aligned}
& \dot{x}(t)=A(t) \cdot x(t)+B(t) \cdot u(t) \\
& y(t)=C(t) \cdot x(t)+D(t) \cdot u(t)
\end{aligned}
$$

where $x(t) \in \Re^{n}$ represents the state vector, $u(t) \in \Re^{m}$ is the input vector and $y(t) \in \Re^{p}$ is the output vector. The $A(t) \in$ $\Re^{n \times n}$ is the state matrix, $B(t) \in \Re^{n \times m}$ is the input matrix, $C(t) \in \Re^{p \times n}$ is the output matrix and $D(t) \in \Re^{p \times m}$ is the forward matrix. When a process is dynamically changing in time, the values of the different vectors and matrices described in (1) are changing as well.

Merging the matrices into one single matrix:

$$
S(t)=\left(\begin{array}{ll}
A(t) & B(t) \\
C(t) & D(t)
\end{array}\right)
$$

where $S(t) \in \Re^{(n+p) \times(n+m)}$, (1) picks up the following simpler form:

$$
\left(\begin{array}{l}
\dot{x}(t) \\
y(t)
\end{array}\right)=S(t)\left(\begin{array}{l}
x(t) \\
u(t)
\end{array}\right)
$$

On the other hand, the general representation of a LPV model can be described as follows [3]:

$$
\begin{aligned}
& \dot{x}(t)=A(\rho(t)) \cdot x(t)+B(\rho(t)) \cdot u(t) \\
& y(t)=C(\rho(t)) \cdot x(t)+D(\rho(t)) \cdot u(t)
\end{aligned}
$$

In this case, the matrices depend on the $\rho(t) \in \Re^{k}$ called as parameter vector or scheduling parameter vector. Now the dependency of the matrices in time comes through the parameter vector's elements, which are time dependent. If the $\rho(t)$ vector contains elements from the $x(t)$ vector, the model is called quasi-LPV (qLPV) model, otherwise its called LPV system. Similarly to (2) the parameter dependent matrices can be described in one compact matrix:

$$
S(\rho(t))=\left(\begin{array}{ll}
A(\rho(t)) & B(\rho(t)) \\
C(\rho(t)) & D(\rho(t))
\end{array}\right)
$$

Using (4) and (5) the qLPV system can be written as:

$$
\left(\begin{array}{l}
\dot{x}(t) \\
y(t)
\end{array}\right)=S(\rho(t))\left(\begin{array}{l}
x(t) \\
u(t)
\end{array}\right)
$$

a compact form to describe parameter dependent and time varying processes.

The current paper focuses on the error interpretation of LPV models in order to efficiently support quality requirements of LPV model related control problems. The structure of the paper is as follows: Section 2 gives a brief overview of LPV representations together with the proposed quality criteria on LPV systems, while Section 3 demonstrates it on a glucoseinsulin model used under intensive care.

\section{LPV CONFIGURATIONS}

There are a few possibilities of representing a LPV system: affine-, polytopic- and TP-configurations [3]-[6].

\section{A. Affine $L P V$ configuration}

In case of affine LPV configuration the matrices of the state-space representation of a system are the affine functions of the scheduling variables, namely, the parameter vector. The parameter dependent matrices consist from constant and varying parts.

$$
\begin{aligned}
& A(\rho(t))=A_{0}+\sum_{i=1}^{n} \rho_{i}(t) \cdot A_{i} \\
& B(\rho(t))=B_{0}+\sum_{i=1}^{n} \rho_{i}(t) \cdot B_{i} \\
& C(\rho(t))=C_{0}+\sum_{i=1}^{n} \rho_{i}(t) \cdot C_{i} \\
& D(\rho(t))=D_{0}+\sum_{i=1}^{n} \rho_{i}(t) \cdot D_{i}
\end{aligned}
$$


Similarly to (2) and (5) the compact matrix is as follows:

$$
S(\rho(t))=\left(\begin{array}{ll}
A_{0}+\sum_{i=1}^{n} \rho_{i}(t) \cdot A_{i} & B_{0}+\sum_{i=1}^{n} \rho_{i}(t) \cdot B_{i} \\
C_{0}+\sum_{i=1}^{n} \rho_{i}(t) \cdot C_{i} & D_{0}+\sum_{i=1}^{n} \rho_{i}(t) \cdot D_{i}
\end{array}\right)
$$

The constant and time varying parts can be highlighted as:

$$
\begin{gathered}
S_{0}=\left(\begin{array}{cc}
A_{0} & B_{0} \\
C_{0} & D_{0}
\end{array}\right), \\
\sum_{i=1}^{n} \rho_{i}(t) \cdot S_{i}=\left(\begin{array}{ll}
\sum_{i=1}^{n} \rho_{i}(t) \cdot A_{i} & \sum_{i=1}^{n} \rho_{i}(t) \cdot B_{i} \\
\sum_{i=1}^{n} \rho_{i}(t) \cdot C_{i} & \sum_{i=1}^{n} \rho_{i}(t) \cdot D_{i}
\end{array}\right)
\end{gathered}
$$

Hence, the affine parameter dependent compact matrix is:

$$
S(\rho(t))=S_{0}+\sum_{i=1}^{n} \rho_{i}(t) \cdot S_{i}
$$

while the simpler affine LPV system form is as follows:

$$
\left(\begin{array}{l}
\dot{x}(t) \\
y(t)
\end{array}\right)=S(\rho(t))\left(\begin{array}{l}
x(t) \\
u(t)
\end{array}\right)
$$

In the discrete time domain, the description of the above mentioned definitions become:

$$
S(\rho(k))=S_{0}+\sum_{i=1}^{n} \rho_{i}(k) \cdot S_{i}
$$

where $k$ represents the different discrete timeframes. Hence, the discrete LPV affine system can be described as follows:

$$
\left(\begin{array}{c}
x(k+1) \\
y(k)
\end{array}\right)=S(\rho(k))\left(\begin{array}{l}
x(k) \\
u(k)
\end{array}\right)
$$

\section{B. Parameter Space of Affine System}

The elements of the $\rho \in \Re^{N}$ parameter vector creates the parameter space, i.e. an abstract, arbitrary space with $\operatorname{dim} N$. Hence, the LPV system is determined via the minimum and maximum values of the elements of the parameter vector. This constraint forms a "parameter box" in the parameter space that gives a suitable configuration for control engineering with three classical interpretations:

- The borders of the parameter box represents a reduced "work space", where the controller design becomes easier, since it is enough to investigate the behavior of the system in this well-delimited space;

- In case of uncertain systems, the border of the parameter box can be represented by the minimum and maximum of the selected parameter uncertainty;
- The affine representation is mainly used to hide a original nonlinear system's nonlinearity, allowing the use of linear control theory on the direct nonlinear system itself.

Figure 1 visualizes a $3 D$ parameter space representation of an affine LPV systems $\left(\rho \in \Re^{3}\right)$, with time varying parameters between given minimum and maximum values:

$$
\rho(t)=\left(\begin{array}{l}
\rho_{1}(t) \\
\rho_{2}(t) \\
\rho_{3}(t)
\end{array}\right)=\left(\begin{array}{l}
{\left[\rho_{1}^{-} . . \rho_{1}^{+}\right]} \\
{\left[\rho_{2}^{-} . . \rho_{2}^{+}\right]} \\
{\left[\rho_{3}^{-} . . \rho_{3}^{+}\right]}
\end{array}\right)
$$

\section{Polytopic LPV configuration}

If affine representation is not possible, polytopic configuration is used, where the LPV vertex is created by the linear combination of linearized systems in stable working points [1].

The barycentric calculus discovered by Möbius in the 19th century, [7], [8] let to describe a point inside a convex triangle by using the vertices. Warren et al. demonstrated the possibility to describe a point inside an arbitrary convex set by using arbitrary vertices of the given set [9]. This method can be used in control engineering applications, as well [3]. The connection between the affine and polytopic LPV systems can be easily identified, since if the parameter box of the affine system can be handled as a convex polytope and every system representation inside this polytope can be described with the convex combination of the vertices of the polytope, then the convexity criteria is satisfied, namely:

1) The polytopic coordinates, $\alpha_{i} \geq 0$ are non-negative

2) The sum of the polytopic coordinates is $\sum_{i=1}^{N} \alpha_{i}=1$

3) The system inside this polytope is equal with the convex combination of the vertices $S=\sum_{i=1}^{N} \alpha_{i} S_{i}$ or in short form [2]:

$$
\left\{S=\sum_{i=1}^{N} \alpha_{i} S_{i}: \alpha_{i} \geq 0, \sum_{i=1}^{N} \alpha_{i}=1\right\}
$$

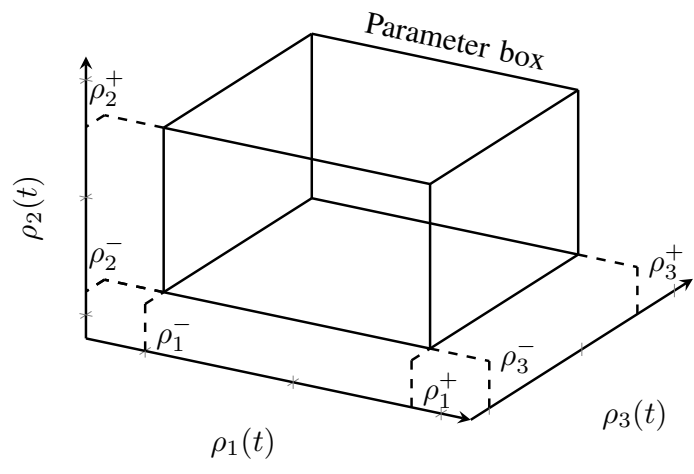

Figure 1: 3D affine parameter representation. 


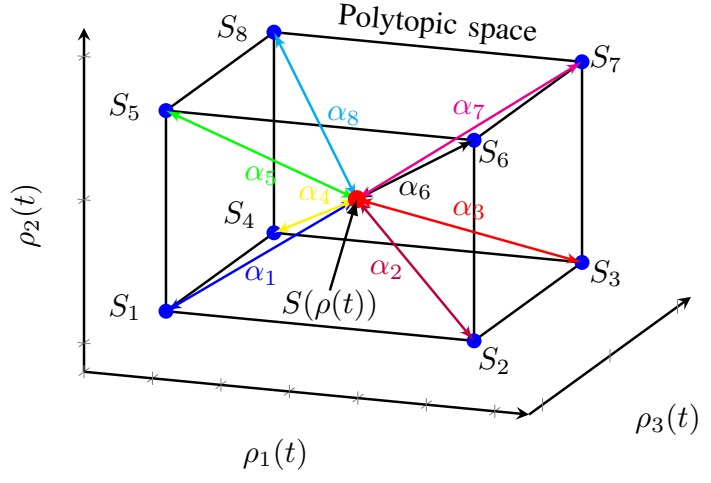

Figure 2: 3D polytopic representation.

There are a few properties of a polytopic LPV representation which need to be cleared. In order to ease its understanding, we present these on a $3 D$ polytopic space (Fig.2). In case of the parameter dependent system $S(\rho(t))$, the parameter dependency will be connected to the $\alpha(\rho(t))$ scalers (as convex coordinates). The vertex of the polytopic parameter space can be interpreted as a Linear Time Inveriant (LTI) system, since, the parameters in the vertex are fixed during operation, the related $\alpha$ coordinate is equal with 1 , while the other polytopic coordinates are equal with zero.

Generally the polytopic model can be described as follows:

$$
S(\rho(t))=\sum_{i=1}^{8} \alpha_{i}(\rho(t)) \cdot S_{i}
$$

if the parameter dependent system is inside the polytope. The actual system can be calculated by barycentric calculus with using the vertices of the complex parameter space, namely, the actual system in the parameter space is the complex combination of the vertices of the given polytope [2], [3], [9].

However, if the system is in the vertex $S_{1}$, then the system description is modified:

$$
\begin{gathered}
S(\rho(t))=\alpha_{1}(\rho(t)) \cdot S_{1}+\sum_{i=2}^{8} \alpha_{i}(\rho(t)) \cdot S_{i}= \\
\alpha_{1}(\rho(t)) \cdot S_{1} \\
\sum_{i=2}^{8} \alpha_{i}(\rho(t)) \cdot S_{i}=0
\end{gathered}
$$

\section{The introduced quality criteria based on LPV configuration}

In control engineering the most common method of controller design for LPV systems represents Linear Matrix Inequality (LMI) based controller design. LMIs represent a powerful mathematical tool [10], where controller design can be achieved after numerically solving feasibility problems without analytical computations. LMI techniques where introduced in control theory by Lyapunov in his epochal dissertation [11]. He proved that the autonomous system described by

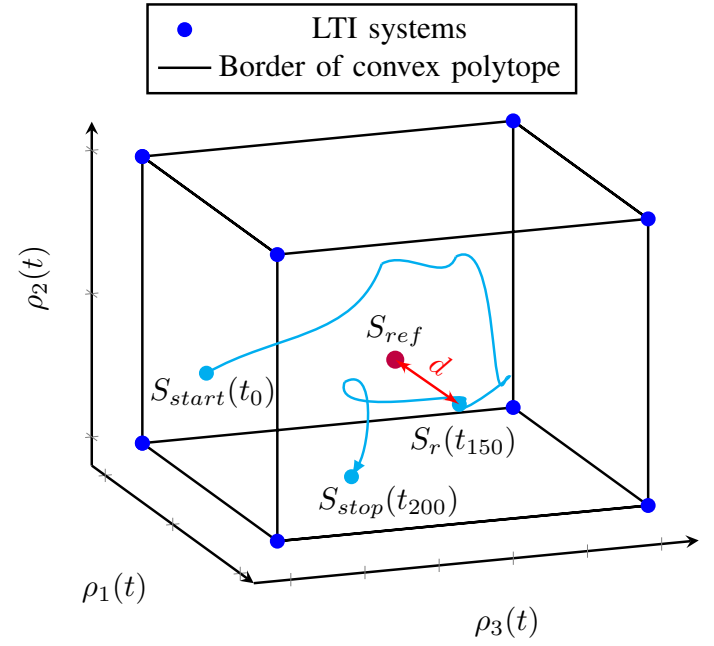

Figure 3: 3 dimensional parameter space $\left(\rho \in \Re^{3}\right)$

$$
\dot{x}(t)=A(t) \cdot x(t)
$$

can be stable, if and only if there is exist a positive definite matrix, $P>0$ such that

$$
A^{T} P+P A<0
$$

Since then, a wide range of different LMI applications were discovered [3], [10], [12]. The basic concept in case of LPVLMI based modeling and control design is the fact that LPV models are valid only inside the convex polytope. Hence, it is enough to find an LMI solution feasible in the vertices of the convex polytope by which a controller can be realized handling every particular LTI system obtained during operation from the original nonlinear LTV system.

The quality and quantity criteria are implemented during controller design. For example, in case of parameter uncertainty an LMI based robust controller can be realized satisfying the output and the performance requirements. The validity analysis of such controllers is mostly based on the eligibility of the requirements, namely, if the obtained controller can deal with the system in the parameter box, the performance criteria is satisfied and the system never exceeds this region then the realized controller is appropriate and can be used for the given application.

Beside the mentioned possibilities a novel quality criteria can be introduced as follows. We illustrate this approach on Fig. 3 for $3 D$ parameter space case (however, this can be extended to arbitrary dimensions as well). The basic idea behind relies on the fact that if we handled the parameter box as a convex polytope, then every point inside can be represented as an LTI system, which can be described as the convex combination of the vertices of the polytope as in (17). Hence, a LPV kind LTV system can be represented as an infinite number of LTI systems which are switching in time at given timeframes from the starting point $S_{\text {start }}$ at $t=0 \mathrm{~min}$ to $S_{\text {stop }}$ (in this given example at $t=200 \mathrm{~min}$ ). 
As a result, every LTI system can be described as the convex combination of the vertices of the polytope, including the reference system as well. That means, a "difference" can be defined, based on the parameter vector of the reference and the operating point. This difference can be interpreted as an error signal between the operating system, $S_{r}\left(t_{i}\right)$ and the predefined, designated reference $S_{r e f}$.

The error signal introduced in this way is a vector evaluated at every moment. For the current paper we only defined two type of error signals beyond the difference itself, as quality criteria:

- Error "vector", which is the difference between the operating system and reference system in the polytope. It can be interpreted similarly as settling time in classical control theory, as a sphere simplex can be defined around the reference system in the space of the polytope, and the time when the operating system's trajectory does not exceed this sphere simplex domain can be measured;

- The 2 norm of the difference also can be used, as a norm based error signal;

- The reference system and the operating system also can be interpreted as "vectors" in the convex polytope together with the scalar dot product; hence, scalar dot based error signal can be introduced.

In the following section we exemplify the previously defined errors on a real case model.

\section{Illustrative Example: the GluCOSE-INSUlin SYSTEM IN ICU}

\section{A. A glucose-insulin model and its LPV configuration}

We selected a simple and well-known model of the glucoseinsulin interaction, which appropriately demonstrates the generation of the introduced performance property.

1) The Canterbury-model: The model was developed in New Zealand at University of Canterbury [13], [14], and originally describse the glucose-insulin dynamics of inpatients in Intensive Care Unit (ICU). It gives a description about the current and prediction about the future metabolic conditions of the patient with sufficient and demonstrated accuracy. The model is listed below:

$$
\begin{aligned}
\dot{G}(t) & =-p_{G} G(t)-S_{I}(t)\left(G(t)+G_{E}\right) . \\
\dot{X}(t) & =-k I(t)-k Q(t) \\
\dot{I}(t) & =-\frac{n I(t)}{1+\alpha_{G} Q(t)}+P(t) \\
\dot{I}(t) & \frac{u_{e x}(t)}{V}
\end{aligned}
$$

The output of the model is the $G(t)[\mathrm{mg} / \mathrm{dL}]$ blood glucose (BG) level, while the input is the $u_{e x}(t)[\mathrm{mU} / \mathrm{min}]$ external insulin and the $P(t)[\mathrm{mmol} / \mathrm{L} / \mathrm{min}]$ toal plasma glucose input. $G(t)$ is considered state as well, together with the concentration of insulin bounded to interstitial sites $Q(t)[m U / L]$ and the plasma inulin resulting from external input $I(t)[m U / L]$. The parameters are the $G_{E}=10.5[\mathrm{mmol} / L]$ plasma equilibrium level, $p_{G}=0.01[1 / \mathrm{min}]$ endogenous glucose clearence, $S_{I}=0.001[\mathrm{~L} / \mathrm{mU} / \mathrm{min}]$ insulin sensitivity, $V=12[L]$ insulin distribution volume, $k=0.0198[1 / \mathrm{min}]$ effective life of insulin in the compartment, $n=0.16[1 / \mathrm{min}]$ first order decay rate from plasma, $\alpha_{I}=0.0017[L / m U]$ plasma insulin disappearance and $\alpha_{G}=0.0154[L / m U]$ insulin effect.

2) $q L P V$ model of the Canterbury-model: The selected parameter vector was the following:

$$
\rho(t)=\left[\begin{array}{c}
\rho_{1}(t) \\
\rho_{2}(t) \\
\rho_{3}(t)
\end{array}\right]=\left[\begin{array}{c}
\frac{S_{I}(t) Q(t)}{1+\alpha_{G} Q(t)} \\
\frac{S_{I}(t) G_{E}}{1+\alpha_{G} Q(t)} \\
\frac{1}{1+\alpha_{I} I(t)}
\end{array}\right]
$$

As a result, the qLPV model of the Canterbury-model can be described as follows:

$$
\begin{aligned}
& A(\rho(t))=A_{0}+A_{1} \rho_{1}(t)+A_{2} \rho_{2}(t)+A_{3} \rho_{3}(t)= \\
& {\left[\begin{array}{ccc}
-p_{G} & 0 & 0 \\
0 & -k & k \\
0 & 0 & 0
\end{array}\right]+\left[\begin{array}{ccc}
0 & -1 & 0 \\
0 & 0 & 0 \\
0 & 0 & 0
\end{array}\right] \rho_{1}(t)} \\
& +\left[\begin{array}{ccc}
0 & -1 & 0 \\
0 & 0 & 0 \\
0 & 0 & 0
\end{array}\right] \rho_{2}(t)+\left[\begin{array}{ccc}
0 & 0 & 0 \\
0 & 0 & 0 \\
0 & 0 & -n
\end{array}\right] \rho_{3}(t) \\
& B(\rho(t))=\left[\begin{array}{lll}
1 & 0 & 0 \\
0 & 0 & 0 \\
0 & \frac{1}{V_{L}} & -p_{4} I_{b}
\end{array}\right] \\
& C(\rho(t))=\left[\begin{array}{lll}
1 & 0 & 0
\end{array}\right]
\end{aligned}
$$

From this exactly created affine model the polytopic model form can be easily computed after specifying the borders of the parameter box (serving as the vertices of the convex polytope as well). The selected borders and through them the obtained vertices are the following:

$$
\rho(t)=\left(\begin{array}{l}
\rho_{1}(t) \\
\rho_{2}(t) \\
\rho_{3}(t)
\end{array}\right)=\left(\begin{array}{l}
{\left[\rho_{1}^{-} . . \rho_{1}^{+}\right]} \\
{\left[\rho_{2}^{-} . . \rho_{2}^{+}\right]} \\
{\left[\rho_{3}^{-} . . \rho_{3}^{+}\right]}
\end{array}\right)=\left(\begin{array}{c}
{[0 . .0 .5]} \\
{[0.01 . .0 .013]} \\
{[0.94 . .1 .02]}
\end{array}\right)
$$

For example, the vertex one is $\rho_{1}=\left(\begin{array}{c}0 \\ 0.01 \\ 0.94\end{array}\right)$ and similarly, the vertex eight is $\rho_{8}=\left(\begin{array}{c}0.5 \\ 0.01 \\ 0.94\end{array}\right)$.

Figure 4 captures the open-loop simulation of the original nonlinear and the polytopic LPV model. It can be seen that the two models are perfectly matching each other in case of an arbitrary input (used here as a pulse generator with amplitude 40 and $150[\mathrm{~min}]$ pulse distance). 

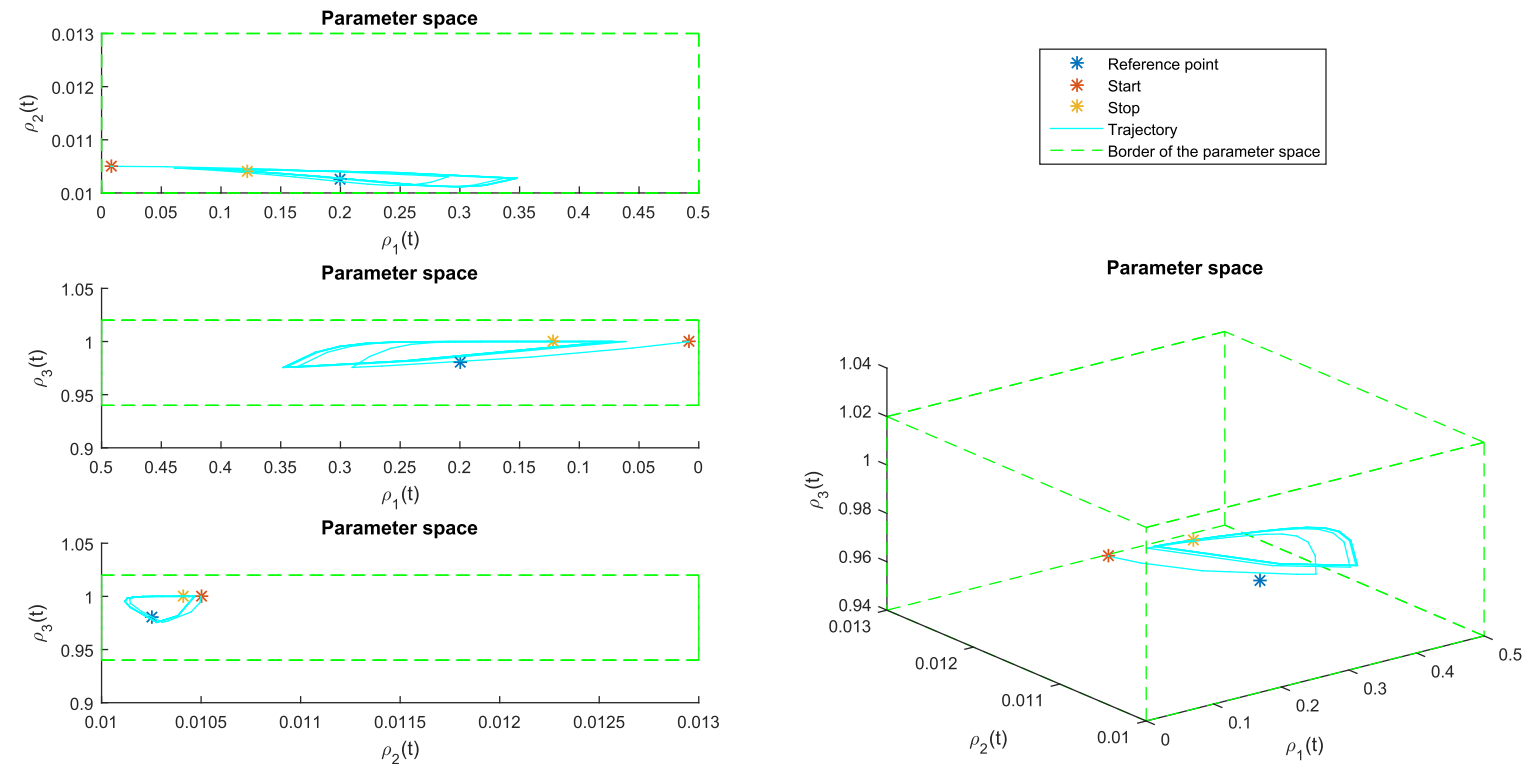

Figure 5: 3D parameter space of the scheduling parameters.
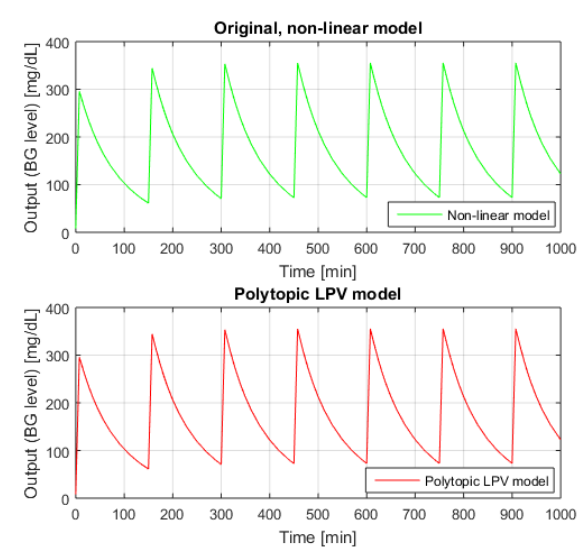

Figure 4: Outputs of the original and polytopic-transformed Canterbury-model.

\section{B. Results}

We selected as reference system the $S_{\text {ref }}=$ $[0.2,0.01025,0.98]^{T}$. Figure 6 presents the changing of the variable of the parameter vector in time. The dashed lines represent the $S_{\text {ref }}$ system in the parameter space. Because of the pulse input generators, after the first impulse the parameters of the system picked up a symmetrical movement meaning that the same LTI systems were obtained periodically over time. This attitude can be seen on Fig.5 as well, where the whole $3 D$ polytope itself is presented. After
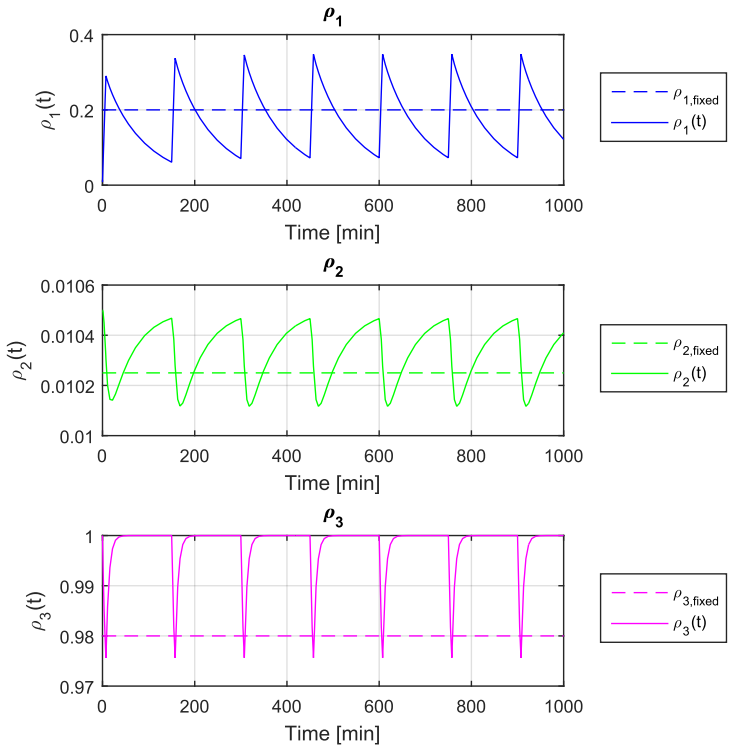

Figure 6: Changing of the scheduling parameters over time.

the first period the system runs trough almost the same path over and over again. The operating system does not meet the reference system during operation which means the existence of an error all the time. In this study, we investigated the norm error and the scaler dot error (Fig.7). 

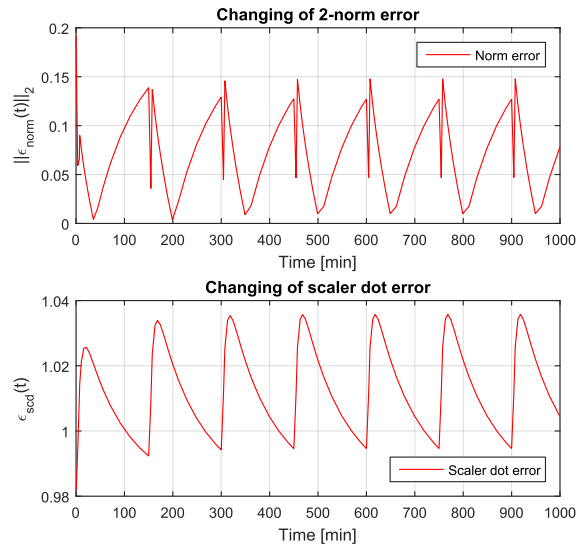

Figure 7: Defined error types.

Table I: Error at different time points

\begin{tabular}{|c|c|c|c|}
\hline & $S_{\text {ref }}$ & $S_{r}\left(t_{154.6}\right)$ & $S_{r}\left(t_{457.5}\right)$ \\
\hline$\rho_{1}$ & 0.2 & 0.2356 & 0.1002 \\
\hline$\rho_{2}$ & 0.01025 & 0.01025 & 0.01044 \\
\hline$\rho_{3}$ & 0.98 & 0.9818 & 1 \\
\hline \multicolumn{4}{|c|}{} \\
\hline & 2-norm error & $\mathbf{0 . 0 3 5 7 4}$ & $\mathbf{0 . 1 4 7 5}$ \\
\hline & Scalar dot error & $\mathbf{1 . 0 0 9}$ & $\mathbf{1 . 0 2 6}$ \\
\hline
\end{tabular}

The norm error is an absolute error; hence, if the reference system and the operating system is the same then the norm error will be equal with 0 . In contrary, the scalar dot error produces 1 as the systems' parameters are equal.

We highlighted in the Table I two different points from the parameter space at different timeframes, as are good representation of the introduced error interpretation. The small values of the parameters come from the properties of the model, another selection of the variable in the parameter vector may produces higher values. In the first case at $154.6 \mathrm{~min}$, the operating system and the reference system are close to each other so the errors are low, while at $457.5 \mathrm{~min}$ the systems are far from each other; hence, the errors are higher.

\section{Conclusion And Future Work}

In this study we introduced a new error interpretation regarding to LPV systems, based on the convex property of the LPV polytope. We have defined how to use these interpretations as quality criteria during control design.

Future work will focus on the investigation of how this appraoch can be implemented to the actual LMI based control design methods in order to realize more precise controller for the practice.

\section{ACKNOWLEDGMENT}

Gy. Eigner thankfully acknowledge the support of the Robotics Special College of Obuda University. L. Kovács is also supported by the János Bolyai Research Scholarship of the Hungarian Academy of Sciences. The research was also supported by the Research and Innovation Center of Obuda University.

\section{REFERENCES}

[1] O. Sename, P. Gáspár, and J. Bokor, Eds., Robust Control and Linear Parameter Varying Approaches, Application to Vehicle Dynamics, 1 st ed., ser. Lecture Notes in Control and Information Sciences. Berlin: Springer-Verlag, 2013.

[2] D. Gu, P. Petkov, and M. Konstantinov, Robust Control Design with Matlab, 2nd ed. London: Springer, 2013.

[3] A. White, G. Zhu, and J. Choi, Linear Parameter Varying Control for Engineering Applicaitons, 1st ed. London: Springer, 2013.

[4] C. Scherer and S. Weiland, "Lecture notes disc course on linear matrix inequalities in control," Delft University, 1999.

[5] B. Takarics and P. Baranyi, "Tp model-based robust stabilization of the 3 degrees-of-freedom aeroelastic wing section," ACTA Polytech Hung, vol. 12 , no. 1 , pp. $209-228,2015$.

[6] - "Friction compensation in tp model form - aeroelastic wing as an example system," ACTA Polytech Hung, vol. 12, no. 4, pp. 127 - 145, 2015.

[7] F. Möbius, The Barycentric Calculus (in German), 1st ed. Leipzig: Verlag von Johann Ambrosius Barth, 1827.

[8] J. Fauvel, F. Raymond, and R. Wilson, Eds., Möbius and his Band, 1st ed. Oxford: Oxford University Press, 1993.

[9] J. Warren, S. Schaefer, A. Hirani, and M. Desbrun, "Barycentric coordinates for convex sets," Adv Comput Math, vol. 27, pp. 319-338, 2007.

[10] S. Boyd, L. El Ghaoui, E. Feron, and V. Balakrishnan, Linear Matrix Inequalities in System and Control Theory, 1st ed., ser. SIAM Studies in Applied Mathematics. Philadelphia, Pennsylvania, USA: SIAM, 1994.

[11] A. Lyapunov, "The general problem of the stability of motion (translated by a. t. fuller, london, taylor francis)," Automatica, vol. 3, no. 2, pp. $353-356,1995$.

[12] C. Scherer and S. Weiland, Linear Matrix Inequalities in Control, ser. Lecture Notes. Delft: Delft Center for Systems and Control, 2004.

[13] X. Wong, J. Chase, G. Shaw, C. Hanna, T. Lotz, J. Lin, I. Singh-Levett, L. Hollingsworth, and O. Wong, "Model predictive glycaemic regulation in critical illness using insulin and nutrition input: A pilot study," Med Eng Phys, vol. 28, pp. 665 - 681, 2006.

[14] X. Wong, J. Chase, G. Shaw, C. Hann, J. Lin, and T. Lotz, "Comparison of adaptive and sliding-scale glycaemic control in critical care and the impact of nutritional inputs," in 12th International Conference On Biomedical Engineering, p. 4. 